\title{
Highly Efficient White Organic Light-Emitting Diodes with Controllable Excitons Behavior by a Mixed Interlayer between Fluorescence Blue and Phosphorescence Yellow-Emitting Layers
}

\author{
Chun-Hong Gao, Xiao-Bo Shi, Dong-Ying Zhou, Lei Zhang, \\ Zhao-Kui Wang, and Liang-Sheng Liao
}

Jiangsu Key Laboratory for Carbon-Based Functional Materials \& Devices, Institute of Functional Nano \& Soft Materials (FUNSOM), Soochow University, Suzhou, Jiangsu 215123, China

Correspondence should be addressed to Zhao-Kui Wang; zhaokuiwang@hotmail.com and Liang-Sheng Liao; 1sliao@suda.edu.cn Received 7 January 2013; Revised 18 March 2013; Accepted 19 March 2013

Academic Editor: K. N. Narayanan Unni

Copyright (C) 2013 Chun-Hong Gao et al. This is an open access article distributed under the Creative Commons Attribution License, which permits unrestricted use, distribution, and reproduction in any medium, provided the original work is properly cited.

\begin{abstract}
A highly efficient hybrid white organic light-emitting diode (HWOLED) has been demonstrated with a mixed interlayer between fluorescent blue and phosphorescent yellow-emitting layers. The device structure is simplified by using a controllable fluorescencemixed interlayer-phosphorescence emission layer structure. The electroluminance (EL) performance can be modulated easily by adjusting the ratio of the hole-predominated material to the electron-predominated material in the interlayer. It is found that the HWOLED with a ratio of $3: 2$ exhibits a current efficiency of $34 \mathrm{~cd} / \mathrm{A}$ and a power efficiency of $29 \mathrm{~lm} / \mathrm{W}$ at $1000 \mathrm{~cd} / \mathrm{m}^{2}$ with warm white Commission Internationale de l'Eclairage (CIE1931) coordinates of $(0.4273,0.4439)$. The improved efficiency and adaptive CIE coordinates are attributed to the controllable mixed interlayer with enhanced charge carrier transport, optimized excitons distribution, and improved harvestings of singlet and triplet excitons.
\end{abstract}

\section{Introduction}

Since firstly reported by Tang and Vanslyke in 1980s [1], organic light-emitting diodes (OLEDs) especially the white OLEDs (WOLEDs) [2] have drawn increasing attention for wide commercial applications in displays and solid-state lighting, owing to their thinness, flexibility, low operation voltage, wide-viewing angle, high resolution, and a fast response time.

Several kinds of structures have been developed for the WOLED fabrications, such as single emitting layer (EML) [3-5], multi EML [6, 7], blue EML with down conversion layer [8], and tandem structures [9-12] employing fluorescent and/or phosphorescent emissive materials. Phosphorescent materials are the outstanding candidates because of their potential for almost $100 \%$ internal quantum efficiency [13]. However, few blue phosphorescent materials could be considered as an appropriate candidate for WOLEDs until now owing to their short lifetime and high energy gap which causes difficulties in keeping color stability and in finding suitable host materials. Hybrid WOLEDs (HWOLEDs) with an architecture of fluorescence blueinterlayer-phosphorescence complementary color (s)-interlayer-fluorescence blue (F-I-P-I-F), proposed by Sun et al. in 2006 [14], have been paid increasing attention due to the long-term stability of fluorescence blue materials and the long lifetime of the color complementary phosphorescent dopants.

The interlayer between fluorescent blue-emitting layer (EML) and complementary phosphorescent EML plays an important role in the efficiency, brightness, and lifetime since it prevents the singlet energy transfer from the blue fluorescent emitter to the complementary phosphorescent emitters and minimizes the exchange energy losses. Various organic materials with hole-predominated property [15-17], electronpredominated property [18], ambipolar properties [19-22], and mixed interlayer (MI) with hole-predominated material and electron-predominated material [23-32] have been used as the interlayer. The MI is a simple way to effectively 
balance carrier transporting property to achieve improved EL performance in the devices [25-31]. However, the functions that the ratio of the hole-predominated material to electronpredominated material of MI plays in the location of recombination zone and the harvest mechanism of singlet and triplet excitons are not clear enough. Therefore, systematic investigation in order to achieve a suitable MI is still needed. In this study, we investigate the effects of the ratio of the hole-predominated material to the electron-predominated material in MI on the device efficiency and the white color coordinates in HWOLEDs. In addition, we employed an optimized emitting architecture of fluorescence blue-MIphosphorescence yellow (F-MI-P) instead of the generally used F-I-P-I-F architecture [14, 20-22] for simplifying the fabrication processes. The blue fluorescence and yellow phosphorescence, instead of conventional doping in the ambipolar host and/or the mixed bipolar property host materials [20$24,26-28,30,31]$, are doped in the hole-predominated host and the electron-predominated host, respectively.

\section{Experimental Section}

All layers were fabricated on precleaned and $15 \mathrm{~min}$ UVozone-treated indium-tin-oxide (ITO) electrode on a glass substrate. The thickness and the sheet resistance of the ITO electrode were $110 \mathrm{~nm}$ and $15 \Omega / \square$, respectively. The organic and metallic layers were subsequently deposited by thermal evaporation under a base pressure of $2 \times$ $10^{-6}$ Torr without breaking vacuum. The materials used in this work include molybdenum trioxide $\left(\mathrm{MoO}_{3}\right)$ used as both a hole injection material and a $p$-type doping material in hole-transporting layer (HTL). 4,4',4" -Tri(Ncarbazolyl)triphenylamine (TCTA) is used as both HTL and exciton-blocking layer (EBL). 4,4'-Bis(carbazol-9yl)biphenyl (CBP) is used as both host and interlayer spacer. $\quad 2^{\prime}, 2^{\prime \prime}$-(1,3,5-Benzinetriyl)-Tris(1-phenyl-1-H-benzimidazole) (TPBi) is used as host, interlayer spacer, and electron transport layer (ETL). 4,4'-Bis(9-ethyl-3carbazovinylene)-1,1'-biphenyl (BCzVBi) is used as a blue fluorescent dopant. Iridium(III)bis(4-phenylthieno[3,2c]pyridinato- $\mathrm{N}, \mathrm{C}^{2^{\prime}}$ )acetylacetonate (PO-01) is used as a yellow phosphorescent dopant. The molecular structures of the organic materials are shown in Figure 1(a). The device structure of the HWOLEDs consists of ITO/MoO $(10 \mathrm{~nm}) / \mathrm{TcTa}: \mathrm{MoO}_{3} 20 \mathrm{vol} . \%(65 \mathrm{~nm}) / \mathrm{TcTa}(10 \mathrm{~nm}) / \mathrm{CBP}$ : BCzVBi 5 vol.\% (10 nm)/Spacer $(5 \mathrm{~nm}) / \mathrm{TPBi}:$ PO-01 3 vol.\% $(20 \mathrm{~nm}) / \mathrm{TPBi}(10 \mathrm{~nm}) / \mathrm{TPBi}$ :Li 0.8 vol.\% $(20 \mathrm{~nm}) / \mathrm{Al}(110$ $\mathrm{nm}$ ), where "Spacer" stands for CBP only (Device A), TPBi only (Device B), and CBP:TPBi with varied ratio of $3: 2$ (Device C), 1:1 (Device D), and 2:3 (Device E), respectively. The lithium-doped TPBi layer $[10,33]$ is used as both an electron injection layer (EIL) and an electron-transporting layer (ETL). The intrinsic TcTa is used as both an electronblocking layer (EBL) and an exciton-blocking layer, while $10 \mathrm{~nm}$ intrinsic TPBi is used as both a self-hole-blocking layer (HBL) and a self-triplet exciton-blocking layer. The schematic diagram of device structures and the energy diagram is shown in Figure 1(b).
The layer thickness and the deposition rate of the materials were monitored in situ using an oscillating quartz thickness monitor. A photometer (Photo Research SpectraScan PR 655) was used to measure the electroluminescent (EL) spectra and Commission Internationale de I'Eclairage (CIE) coordinates of the devices. Combined with a constant current source (Keithley 2400 SourceMeter), the currentvoltage characteristic was measured simultaneously. An optical microscope was used to calculate the active area of each device $\left(0.1 \mathrm{~cm}^{2}\right)$ for reducing possible device-to-device error.

\section{Results and Discussion}

The interfacial energy barrier between emitting layers is critical to the driving voltage of WOLEDs. The current density-voltage $(J-V)$ characteristics shown in Figure 2 exhibit that the driving voltage of HWOLEDs with intrinsic interlayers as spacers (Devices A and B) is higher than that of devices with MIs as spacers (Devices C, D and E) at the same current density. It is reported that a mixed layer of two materials has an intervenient energy level between those of two materials $[26,34]$. In the present work, the lowest unoccupied molecular orbital (LUMO) levels of CBP and TPBi are -2.5 and $-2.8 \mathrm{eV}$, and the highest occupied molecular orbital (HOMO) levels of CBP and TPBi are -5.9 and $-6.3 \mathrm{eV}$, respectively. Therefore, the mixed layer of CBP and TPBi is expected to have suitable LUMO and HOMO levels which can reduce interfacial energy barrier for holes and electrons injection in HWOLEDs with MIs.

The ratio of CBP to TPBi in the spacer is critical to the exciton generation interfaces. The EL spectra of the HWOLEDs at $5 \mathrm{~mA} / \mathrm{cm}^{2}$ in Figure 3 exhibit that the yellow emission intensity is decreasing with the ratio of CBP to TPBi ordered as $1: 0,3: 2,1: 1,2: 3$, and $0: 1$, while the blue emission intensity is increasing with the proportion of CBP decreasing.

Generally, CBP is used as an ambipolar charge transporting material with a predominated-hole-transporting property because its hole mobility $\left(2 \times 10^{-3} \mathrm{~cm}^{2} / \mathrm{V} \mathrm{s}\right)$ is ten times its electron mobility $\left(2 \times 10^{-4} \mathrm{~cm}^{2} / \mathrm{V} \mathrm{s}\right)$ [35]. In this work, electrons cannot pass through well because the LUMO of CBP is higher than the LUMO of adjacent TPBi layer. Therefore, in Device A, electrons are blocked from reaching the blue fluorescent EML (BFEML) by a large energy barrier, the main recombination zone of electron and hole is located at the CBP/yellow phosphorescent EML (YPEML) interface, which results in yellow-dominated emission. Whereas, in Device $\mathrm{B}$, the recombination most likely occurs at the BFEML/TPBi interface. The spacer layer prevents the Förster energy transfer from the BFEML to the yellow emitter, while allowing the diffusion of part of the triplets into the YPEML. Therefore, Device B exhibits mainly blue emission. The experimental results demonstrate that MIs act as a role in optimizing the distribution of excitons generated at both BFEML/MI interface and MI/YPEML interface. And the ratio of the yellow emission intensity to the blue emission intensity can be optimized by adjusting the ratio of CBP to TPBi in MIs.

The spacer plays an important role in the singlet and triplet excitons harvesting as well as in the current efficiency 
<smiles>c1ccc2c(c1)c1ccccc1n2-c1ccc(-c2ccc(-n3c4ccccc4c4ccccc43)cc2)cc1</smiles>

CBP

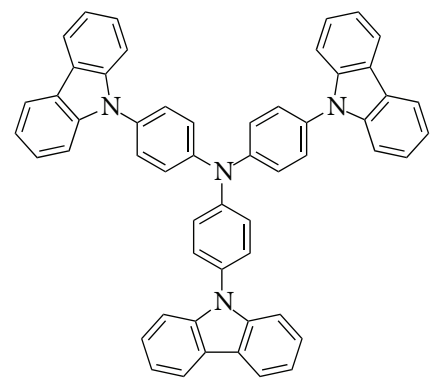

TcTa

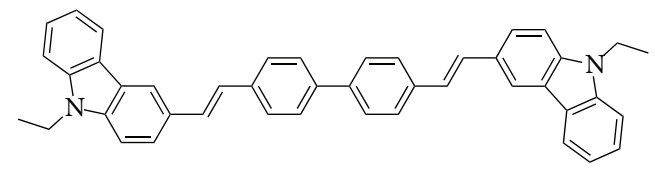

BCzVBi

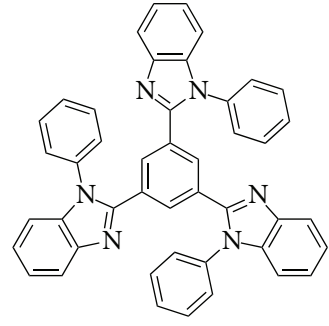

TPBi

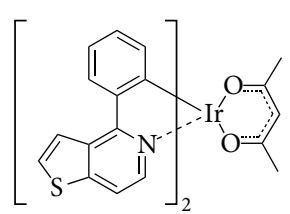

PO-01

(a)
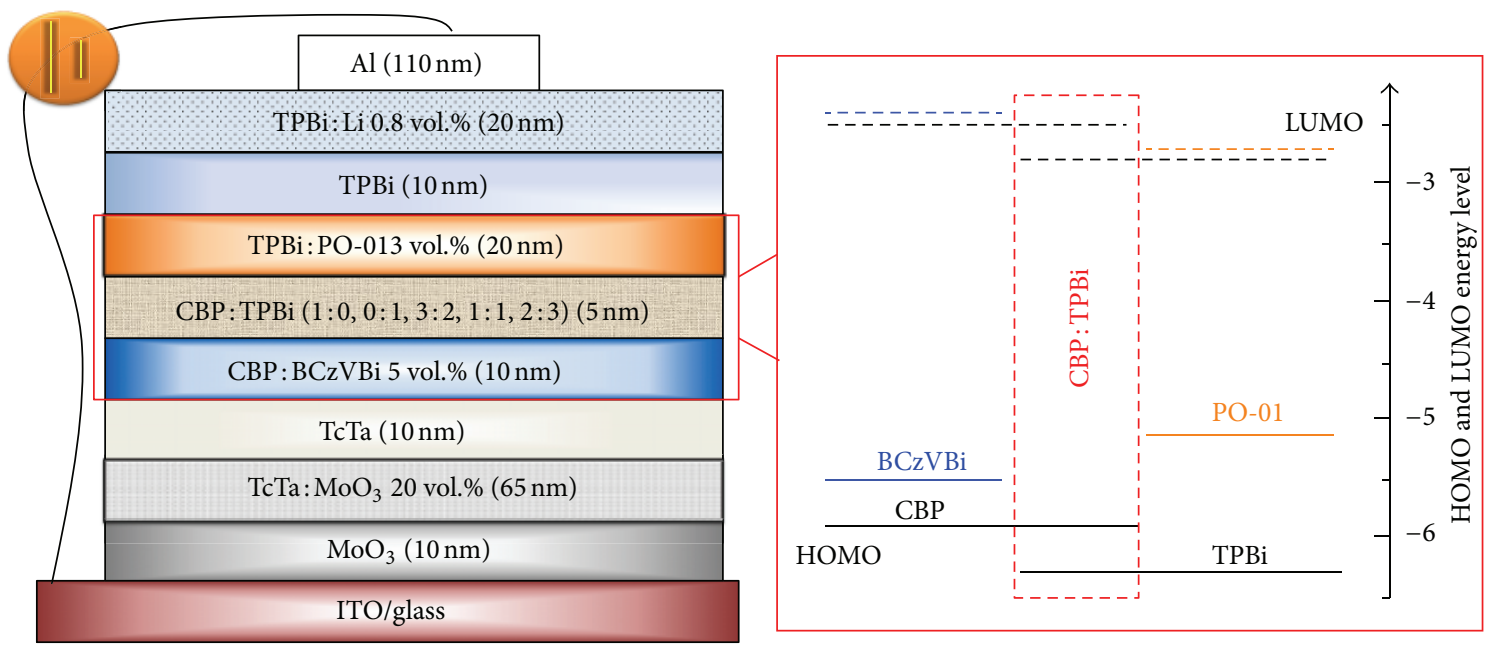

(b)

Figure 1: (a) The chemical structures of organic materials, (b) the device structure and the energy level diagram of HOMO (solid lines) and LUMO (dashed lines) levels (eV) of HWOLEDs (Devices A, B, C, D, and E). The red dashed box depicts the "Spacer" composed of different ratios of CBP to TPBi.

and power efficiency of HWOLEDs. Figures 4(a) and 4(b) show the relationships of current efficiency and power efficiency of the five HWOLEDs under the same current density, respectively. Device A (with intrinsic CBP as spacer) shows the highest efficiency with a maximum current efficiency of $58.5 \mathrm{~cd} / \mathrm{A}$ and a maximum power efficiency of $58.8 \mathrm{~lm} / \mathrm{W}$ at $117 \mathrm{~cd} / \mathrm{m}^{2}$, and $58.4 \mathrm{~cd} / \mathrm{A}, 51.9 \mathrm{~lm} / \mathrm{W}$ with CIE coordinate of $(0.4907,0.5015)$ at $1000 \mathrm{~cd} / \mathrm{m}^{2}$. Conversely, Device B (with intrinsic TPBi as spacer) shows the lowest efficiency with a current efficiency of $6.98 \mathrm{~cd} / \mathrm{A}$ and a power efficiency of $6.09 \mathrm{~lm} / \mathrm{W}$ at $100 \mathrm{~cd} / \mathrm{m}^{2}$, and $6.43 \mathrm{~cd} / \mathrm{A}, 4.5 \mathrm{~lm} / \mathrm{W}$ at $1000 \mathrm{~cd} / \mathrm{m}^{2}$. Furthermore, it demonstrates that both current efficiencies and power efficiencies in the HWOLEDs with
MI spacers are lower than those of Device A and higher than those of Device B, which are strongly dependent on the CBP proportion in MI. The results imply that the harvest of singlet and triplet excitons is crucial in the EL performance. The diffusion length is different for singlet excitons (about several nanometers) and triplet excitons (about several tens of nanometers) [14]. Device A, in which excitons are mainly generated at the CBP/YPEML interface, exhibits the highest efficiency because both singlets and triplets can be harvested by the phosphorescent dopant PO-01, and the triplet Dexter energy transfer from the phosphorescent excitons to the nonradiative triplet state of blue fluorescent emitters is suppressed by the spacer. While Device B, in which excitons 


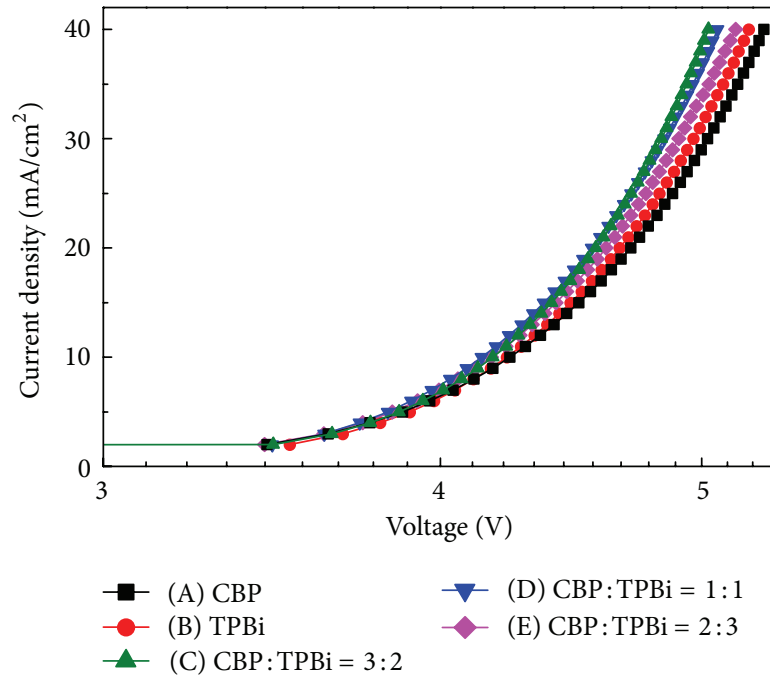

FIgURE 2: The relationship of current density versus voltage in the semilogarithmic scale of HWOLEDs (Devices A, B, C, D, and E).

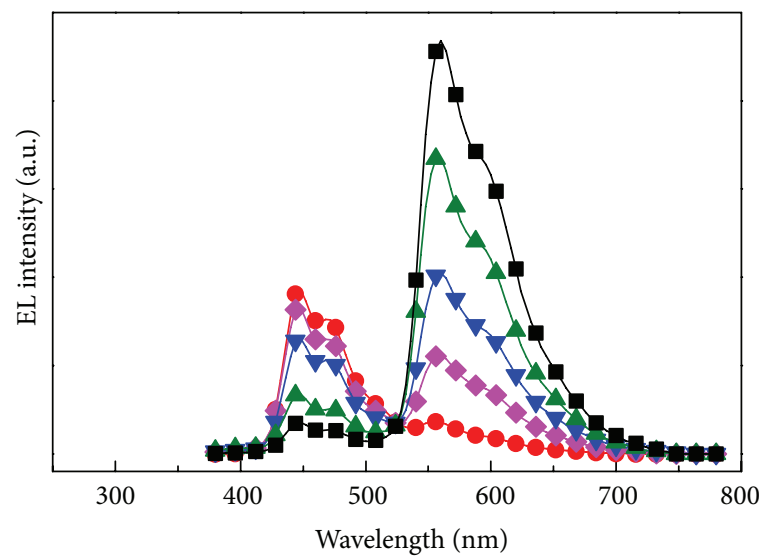

$\begin{array}{ll}- \text { (A) CBP } & - \text { (D) CBP:TPBi }=1: 1 \\ - \text { (B) TPBi } & - \text { (Е) CBP:TPBi }=2: 3 \\ - \text { (C) CBP:TPBi }=3: 2 & \end{array}$

FIGURE 3: The relationship of EL intensity versus wavelength of HWOLEDs (Devices A, B, C, D, and E) at $5 \mathrm{~mA} / \mathrm{cm}^{2}$.

are mainly generated at the BFEML/TPBi interface, exhibits the lowest efficiency since the fluorescent dopant $\mathrm{BCzVBi}$ can only harvest singlets, and most of the triplets are lost via nonradiative process through the low triplet energy level of $\mathrm{BCzVBi}$. In HWOLEDs with $\mathrm{MI}$, excitons are generated at both the interfaces of BFEML/MI and MI/YPEML. At the interface of BFEML/MI, the generated singlet excitons are harvested by the blue fluorescent dopant $(\mathrm{BCzVBi})$ via Förster energy transfer process, and some of the generated triplet excitons can diffuse into the YPEML and are harvested by the yellow phosphorescent dopant (PO-01) via Dexter energy transfer process. Meanwhile, at the interface of MI/YPEML, the generated singlet excitons and triplet excitons are mainly harvested by the yellow phosphorescent

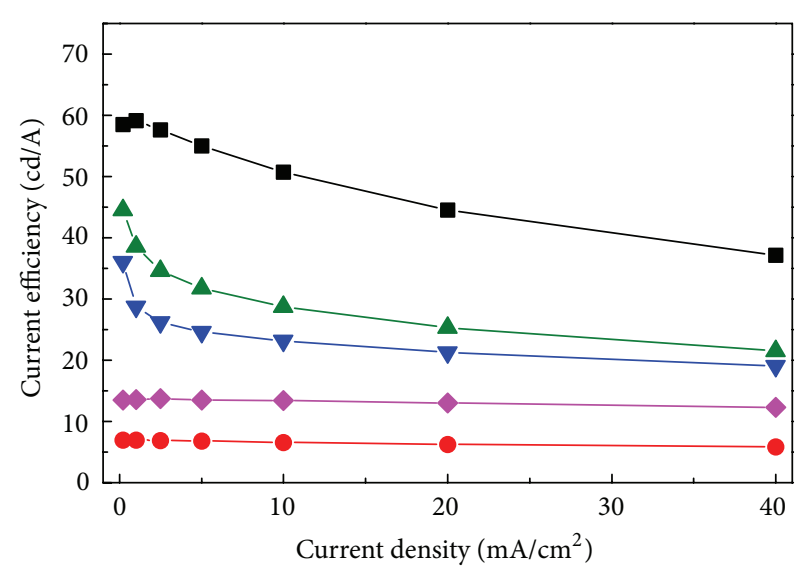

(a)

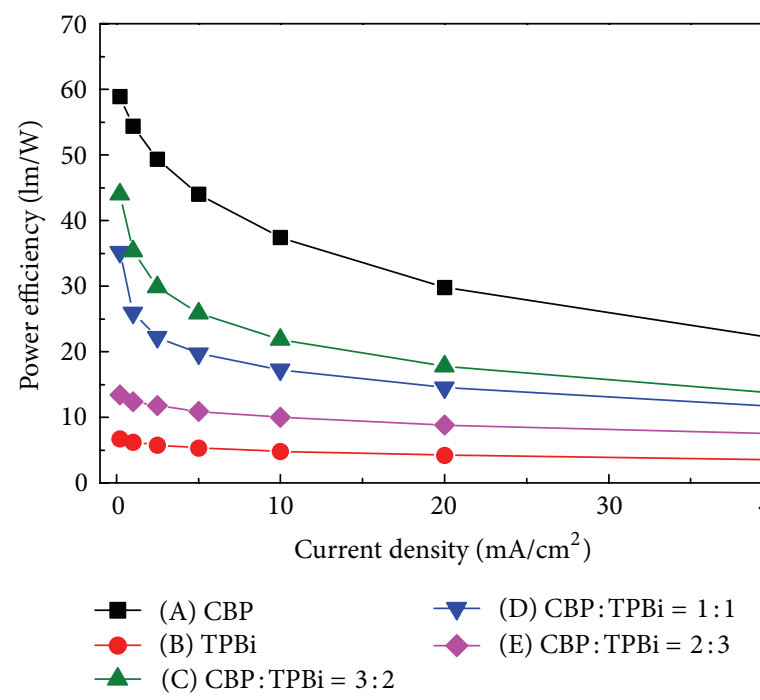

(b)

FIGURE 4: The relationships of (a) current efficiency versus current density and (b) power efficiency versus current density of HWOLEDs (Devices A, B, C, D, and E).

dopant (PO-01) via the intersystem crossing process and the Dexter energy transfer process, respectively.

By adjusting the ratio of CBP to TPBi in MI, both the distribution and the harvest of the singlet and triplet excitons are optimized at both BFEML/MI and MI/YPEML interfaces. As a result, Device $C$ (with a ratio of $3: 2$ ) achieves a warm white emission with a current efficiency of $34.1 \mathrm{~cd} / \mathrm{A}$ and a maximum power efficiency of $29 \mathrm{~lm} / \mathrm{W}$ at $1000 \mathrm{~cd} / \mathrm{m}^{2}$ and corresponding CIE coordinate of $(0.4273,0.4439)$. Particularly, Device D (with a ratio of $1: 1$ ) exhibits a warm white emission with a maximum current efficiency of $36 \mathrm{~cd} / \mathrm{A}$ and a maximum power efficiency of $35.2 \mathrm{~lm} / \mathrm{W}$ at $72 \mathrm{~cd} / \mathrm{m}^{2}$ and corresponding CIE coordinate of $(0.4279,0.4341)$.

The stability of EL spectra is also one of the key factors to evaluate the performance of WOLEDs. Figures 5(a)-5(e) show the EL spectra, normalized by the main emission peak of PO-01 at $560 \mathrm{~nm}$, for HWOLEDs with different spacers at various current densities. The deep blue emission peak 


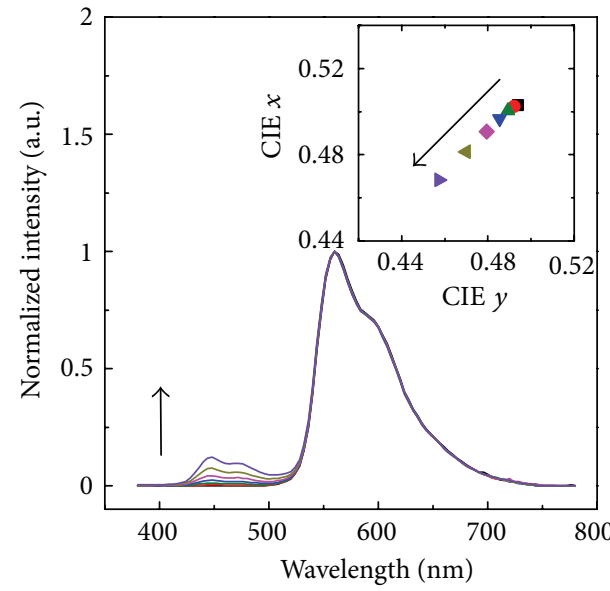

(a) Device A

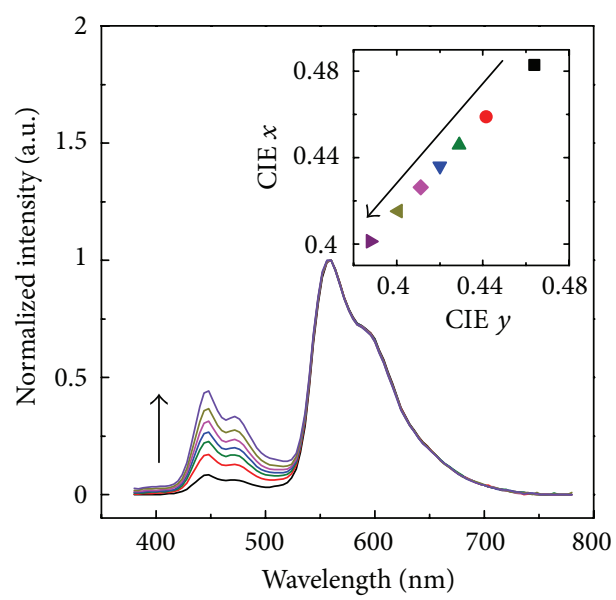

(c) Devices C

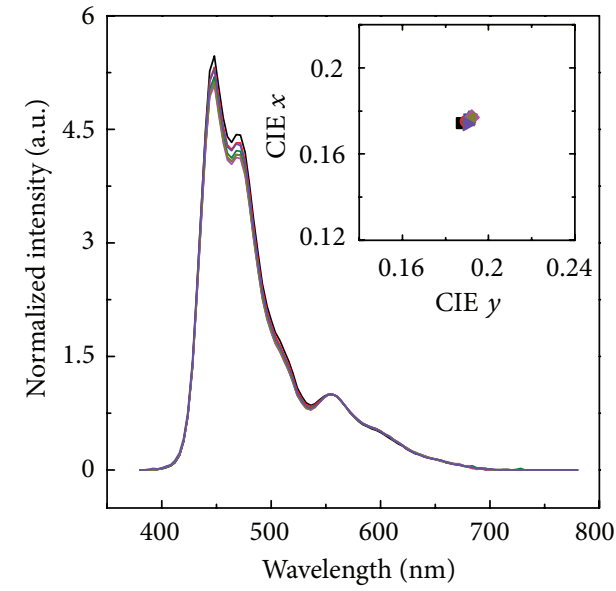

(b) Devices B

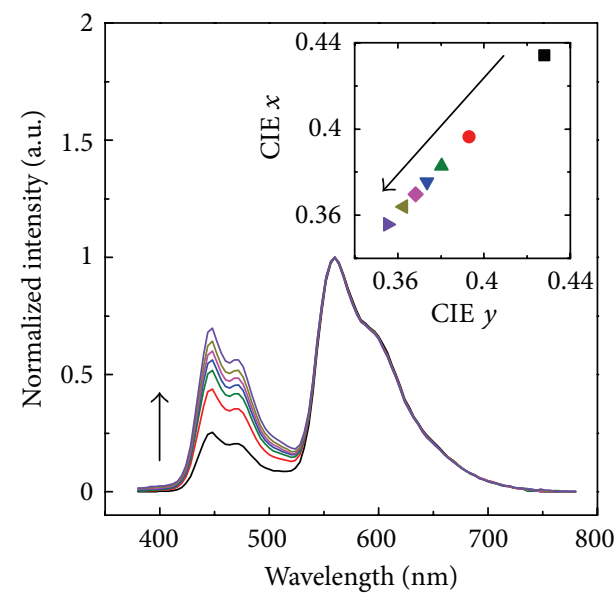

(d) Devices D

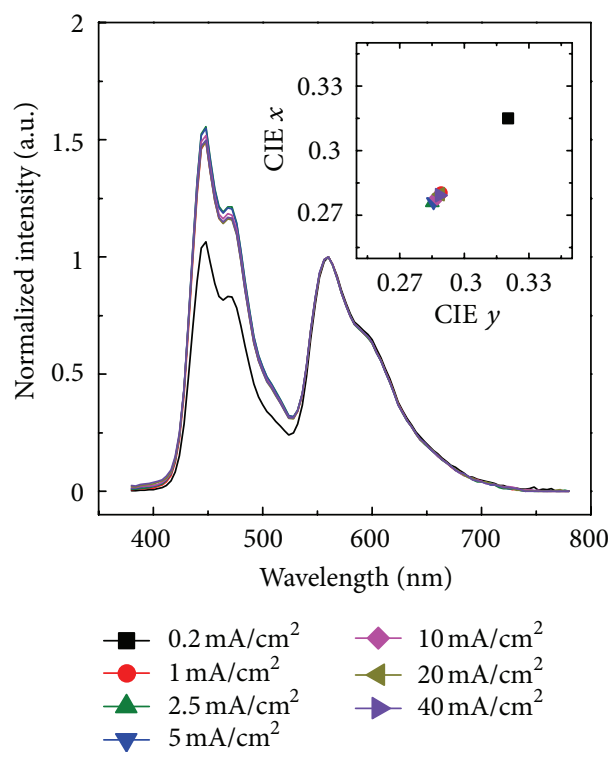

(e) Devices E

FIGURE 5: (a)-(e) Normalized EL spectra of HWOLEDs with different current density. Each inset shows the corresponding CIE coordinates with the same coordinate scale of $\Delta \mathrm{CIE}(0.1,0.1)$. The arrows indicate the EL spectral and CIE color coordinates varying with increasing current density from 0.2 to $40 \mathrm{~mA} / \mathrm{cm}^{2}$. 
$(\sim 448 \mathrm{~nm})$ in the EL spectra shows three different trends in the five HWOLEDs corresponding to different ratios of CBP : TPBi. With increasing current density, the intensity of deep blue emission peak increases in the devices with high CBP proportion (Devices A $(1: 0), C(3: 2)$, and D (1:1)), exhibits small changes in the device without $\mathrm{CBP}$ doping (Device $\mathrm{B}(0: 1)$ ), and shows a rapid increase firstly and then a small change in the device with low CBP proportion (Device $\mathrm{E}(2: 3))$.

With current density increasing, the CIE coordinates exhibit a blue shift in Devices A, C, and D with high CBP proportion. It exhibits a small change of $\triangle \mathrm{CIE}(0.002$, $0.002)$ in Device $B(0: 1)$, and a $\Delta$ CIE $(0.031,0.025)$ shift from $0.2 \mathrm{~mA} / \mathrm{cm}^{2}$ to $1 \mathrm{~mA} / \mathrm{cm}^{2}$ firstly, and then a $\Delta \mathrm{CIE}$ $(0.002,0.002)$ shift in the subsequent applied current density in Device E. The increased blue emission arises from the ambipolar charge transporting properties of $\mathrm{CBP}$ which leads to more electrons injected from TPBi to CBP at an increased current density. As the current density increases, more excitons diffuse to the interface of BFEML/Spacer and cause $\mathrm{BCzVBi}$ emission through Förster energy transfer process, resulting in a blue color shift in Devices A, C and D. This indicates that the recombination region is gradually shifted to the interface of BFEML/Spacer interface from Spacer/YPEML interface in the devices with high CBP proportion when increasing the current density. For Device $\mathrm{B}$, the emission zone is located at the interface of BFEML/TPBi and remains unchangeable when increasing the current density. In Device $\mathrm{E}$ with low CBP proportion, the emission zone is dominated by CBP at low current density and is located at the interface of Spacer/YPEML. The emission zone is gradually dominated by TPBi with increasing current density and shifts to the interface of BFEML/Spacer interface. However, a HWOLED with a stable EL spectrum can be realized by optimizing the ratio of the hole-predominated material (i.e., CBP) to the electron-predominated material (i.e., TPBi) in MI. In the present work, a cool HWOLED with a stable CIE coordinate of $(0.2875 \pm 0.002,0.2782 \pm 0.002)$ is achieved in the luminance range from $136 \mathrm{~cd} / \mathrm{m}^{2}$ to $4916 \mathrm{~cd} / \mathrm{m}^{2}$.

\section{Conclusion}

In summary, we have demonstrated a highly efficient warm white OLED with a simplified F-MI-P structure by optimizing the mixing ratio of hole-predominated material (CBP) and electron-predominated material (TPBi) in MI $(3: 2)$. The influence of the mixing ratio of the hole-predominated material and the electron-predominated material in $\mathrm{MI}$ on the EL performance is investigated. It is found that the improved efficiency with adaptive CIE coordinates is attributed to the enhanced charge carrier transport, optimized excitons distribution at interfaces of both BFEML/MI and MI/YPEML interfaces, and the improved harvest of singlet excitons and triplet excitons, which are modulated by a controllable MI. A cool HWOLED with a stable CIE coordinate off $(0.2875 \pm 0.002,0.2782 \pm 0.002)$ is achieved in the luminance range from $136 \mathrm{~cd} / \mathrm{m}^{2}$ to $4916 \mathrm{~cd} / \mathrm{m}^{2}$ with an optimized mixing ratio of $\mathrm{CBP}: \mathrm{TPBi}=2: 3$ in the MI.

\section{Acknowledgments}

The authors acknowledge financial support from the Natural Science Foundation of China (nos. 21161160446, 61036009, and 61177016), the National High-Tech Research Development Program (no. 2011AA03A110), the Natural Science Foundation of Jiangsu Province (no. BK2010003). This is also a project funded by the Priority Academic Program Development of Jiangsu Higher Education Institutions (PAPD) and by the Research Supporting Program of Suzhou Industrial Park.

\section{References}

[1] C. W. Tang and S. A. Vanslyke, "Organic electroluminescent diodes," Applied Physics Letters, vol. 51, no. 12, pp. 913-915, 1987.

[2] M. C. Gather, A. Kohnen, and K. Meerholz, "White organic light-emitting diodes," Advanced Materials, vol. 23, no. 2, pp. 233-248, 2011.

[3] J. Kido, K. Hongawa, K. Okuyama, and K. Nagai, "White lightemitting organic electroluminescent devices using the poly $(\mathrm{N}$ vinylcarbazole) emitter layer doped with three fluorescent dyes," Applied Physics Letters, vol. 64, no. 7, pp. 815-817, 1994.

[4] Q. Wang, J. Ding, M. Dongge et al., "Harvesting excitons via two parallel channels for efficient white organic LEDs with nearly $100 \%$ internal quantum efficiency: fabrication and emissionmechanism analysis," Advanced Functional Materials, vol. 19, no. 1, pp. 84-95, 2009.

[5] J. Ye, C. J. Zheng, X. M. Ou, X. H. Zhang, M. K. Fung, and C. S. Lee, "Management of singlet and triplet excitons in a single emission layer: a simple approach for a high-efficiency fluorescence/phosphorescence hybrid white organic light-emitting device," Advanced Materials, vol. 24, no. 25, pp. 3410-3414, 2012.

[6] J. Kido, M. Kimura, and K. Nagai, "Multilayer white lightemitting organic electroluminescent device," Science, vol. 267, no. 5202, pp. 1332-1334, 1995.

[7] S. Reineke, F. Lindner, G. Schwartz et al., "White organic lightemitting diodes with fluorescent tube efficiency," Nature, vol. 459, no. 7244, pp. 234-238, 2009.

[8] A. R. Duggal, J. J. Shiang, C. M. Heller, and D. F. Foust, "Organic light-emitting devices for illumination quality white light," Applied Physics Letters, vol. 80, no. 19, pp. 3470-3472, 2002.

[9] L. S. Liao, X. F. Ren, W. J. Begley, Y. S. Tyan, and C. A. Pellow, "Tandem white OLEDs combining fluorescent and phosphorescent emission," SID Symposium Digest of Technical Papers, vol. 39, no. 1, pp. 818-821, 2008.

[10] L. S. Liao, K. P. Klubek, and C. W. Tang, "High-efficiency tandem organic light-emitting diodes," Applied Physics Letters, vol. 84, no. 2, pp. 167-169, 2004.

[11] P. Chen, Q. Xue, W. Xie et al., "Color-stable and efficient stacked white organic light-emitting devices comprising blue fluorescent and orange phosphorescent emissive units," Applied Physics Letters, vol. 93, no. 15, Article ID 153508, 2008.

[12] J. P. Yang, Y. Xiao, Y. H. Deng et al., "Electric-field-assisted charge generation and separation process in transition metal oxide-based interconnectors for tandem organic light-emitting diodes," Advanced Functional Materials, vol. 22, no. 3, pp. 600608, 2012.

[13] M. A. Baldo, D. F. O’Brien, Y. You et al., "Highly efficient phosphorescent emission from organic electroluminescent devices," Nature, vol. 395, no. 6698, pp. 151-154, 1998. 
[14] Y. Sun, N. C. Giebink, H. Kanno, B. Ma, M. E. Thompson, and S. R. Forrest, "Management of singlet and triplet excitons for efficient white organic light-emitting devices," Nature, vol. 440, no. 7086, pp. 908-912, 2006.

[15] G. Schwartz, M. Pfeiffer, S. Reineke, K. Walzer, and K. Leo, "Harvesting triplet excitons from fluorescent blue emitters in white organic light-emitting diodes," Advanced Materials, vol. 19, no. 21, pp. 3672-3676, 2007.

[16] G. Schwartz, S. Reineke, T. C. Rosenow, K. Walzer, and K. Leo, "Triplet harvesting in hybrid white organic light-emitting diodes," Advanced Functional Materials, vol. 19, no. 9, pp. 13191333, 2009.

[17] Q. Wang, C. L. Ho, Y. Zhao, D. Ma, W. Y. Wong, and L. Wang, "Reduced efficiency roll-off in highly efficient and color-stable hybrid WOLEDs: the influence of triplet transfer and chargetransport behavior on enhancing device performance," Organic Electronics, vol. 11, no. 2, pp. 238-246, 2010.

[18] C. L. Ho, M. F. Lin, W. Y. Wong, W. K. Wong, and C. H. Chen, "High-efficiency and color-stable white organic light-emitting devices based on sky blue electrofluorescence and orange electrophosphorescence," Applied Physics Letters, vol. 92, no. 8, Article ID 083301, 2008.

[19] P. Chen, W. Xie, J. Li et al., "White organic light-emitting devices with a bipolar transport layer between blue fluorescent and orange phosphorescent emitting layers," Applied Physics Letters, vol. 91, no. 2, Article ID 023505, 2007.

[20] J. H. Seo, J. H. Seo, J. H. Park et al., "Highly efficient white organic light-emitting diodes using two emitting materials for three primary colors (red, green, and blue)," Applied Physics Letters, vol. 90, no. 20, Article ID 203507, 2007.

[21] J. H. Seo, J. H. Kim, J. H. Seo et al., "Efficient white organic lightemitting diodes by forming major excitons and preventing T-T annihilation," SID Symposium Digest of Technical Papers, p2-38, pp. 1399-1402, 2007.

[22] P. Chen, L. Zhao, Y. Duan et al., "Efficient white organic lightemitting diodes based on an orange iridium phosphorescent complex," Journal of Luminescence, vol. 131, no. 10, pp. 2144-2147, 2011.

[23] K. S. Yook and J. Y. Lee, "Effect of the interlayer composition on the lifetime and color change of hybrid white organic light-emitting diodes," Journal of Industrial and Engineering Chemistry, vol. 17, no. 3, pp. 642-644, 2011.

[24] K. S. Yook, S. O. Jeon, C. W. Joo, and J. Y. Lee, "Color stability and suppressed efficiency roll-off in white organic light-emitting diodes through management of interlayer and host properties," Journal of Industrial and Engineering Chemistry, vol. 15, no. 3, pp. 420-422, 2009.

[25] G. Schwartz, K. Fehse, M. Pfeiffer, K. Walzer, and K. Leo, "Highly efficient white organic light emitting diodes comprising an interlayer to separate fluorescent and phosphorescent regions," Applied Physics Letters, vol. 89, no. 8, Article ID 083509, 2006.

[26] K. S. Yook, S. O. Jeon, C. W. Joo, and J. Y. Lee, "Low driving voltage in white organic light-emitting diodes using an interfacial energy barrier free multilayer emitting structure," Journal of Luminescence, vol. 129, no. 9, pp. 937-940, 2009.

[27] K. S. Yook, S. O. Jeon, C. W. Joo, and J. Y. Lee, "High efficiency, color stability, and stable efficiency roll off in three color hybrid white organic light emitting diodes," Applied Physics Letters, vol. 93, no. 7, Article ID 073302, 2008.
[28] J. H. Seo, I. H. Park, G. Y. Kim et al., "Hybrid spacer for highefficiency white organic light-emitting diodes," Applied Physics Letters, vol. 92, no. 18, Article ID 183303, 2008.

[29] G. Schwartz, S. Reineke, K. Walzer, and K. Leo, "Reduced efficiency roll-off in high-efficiency hybrid white organic lightemitting diodes," Applied Physics Letters, vol. 92, no. 5, Article ID 053311, 2008.

[30] J. H. Seo, J. S. Park, S. J. Lee et al., "Codoped spacer ratio effect of hybrid white organic light-emitting diodes," Current Applied Physics, vol. 11, no. 3, pp. 564-567, 2011.

[31] K. S. Yook, S. O. Jeon, J. Y. Lee et al., "High efficiency pure white organic light-emitting diodes using a diphenylaminofluorenebased blue fluorescent material," Organic Electronics, vol. 10, no. 7, pp. 1378-1381, 2009.

[32] F. Zhao, Z. Zhang, Y. Liu, Y. Dai, J. Chen, and D. Ma, "A hybrid white organic light-emitting diode with stable color and reduced efficiency roll-off by using a bipolar charge carrier switch," Organic Electronics, vol. 13, no. 6, pp. 1049-1055, 2012.

[33] L. S. Liao, W. K. Slusarek, T. K. Hatwar, M. L. Ricks, and D. L. Comfort, "Tandem organic light-emitting diode using hexaazatriphenylene hexacarbonitrile in the intermediate connector," Advanced Materials, vol. 20, no. 2, pp. 324-329, 2008.

[34] S. W. Liu, J. H. Lee, C. C. Lee, C. T. Chen, and J. K. Wang, "Charge carrier mobility of mixed-layer organic light-emitting diodes," Applied Physics Letters, vol. 91, no. 14, 2007.

[35] S. Liu, B. Li, L. Zhang, H. Song, and H. Jiang, "Enhanced efficiency and reduced roll-off in nondoped phosphorescent organic light-emitting devices with triplet multiple quantum well structures," Applied Physics Letters, vol. 97, no. 8, Article ID 083304, 2010. 

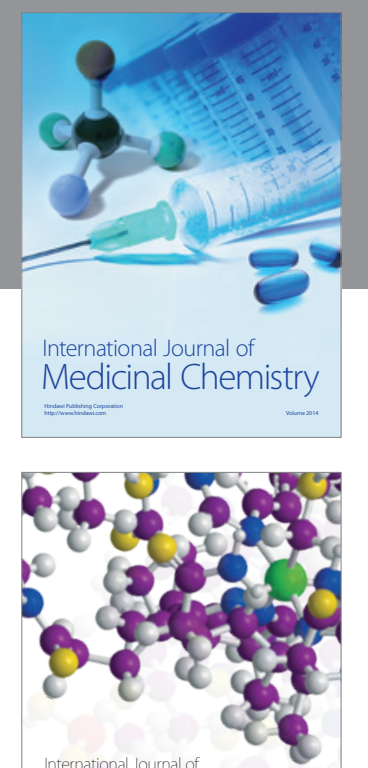

\section{Carbohydrate} Chemistry

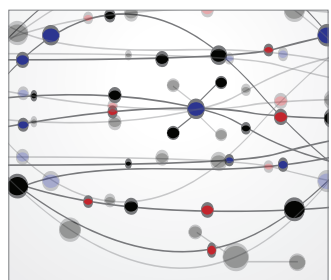

The Scientific World Journal
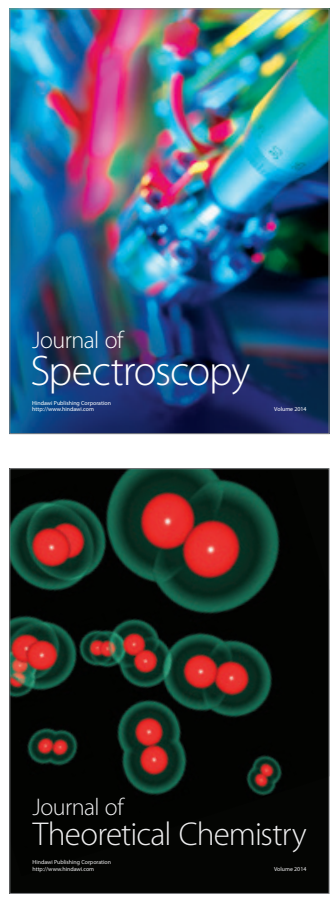
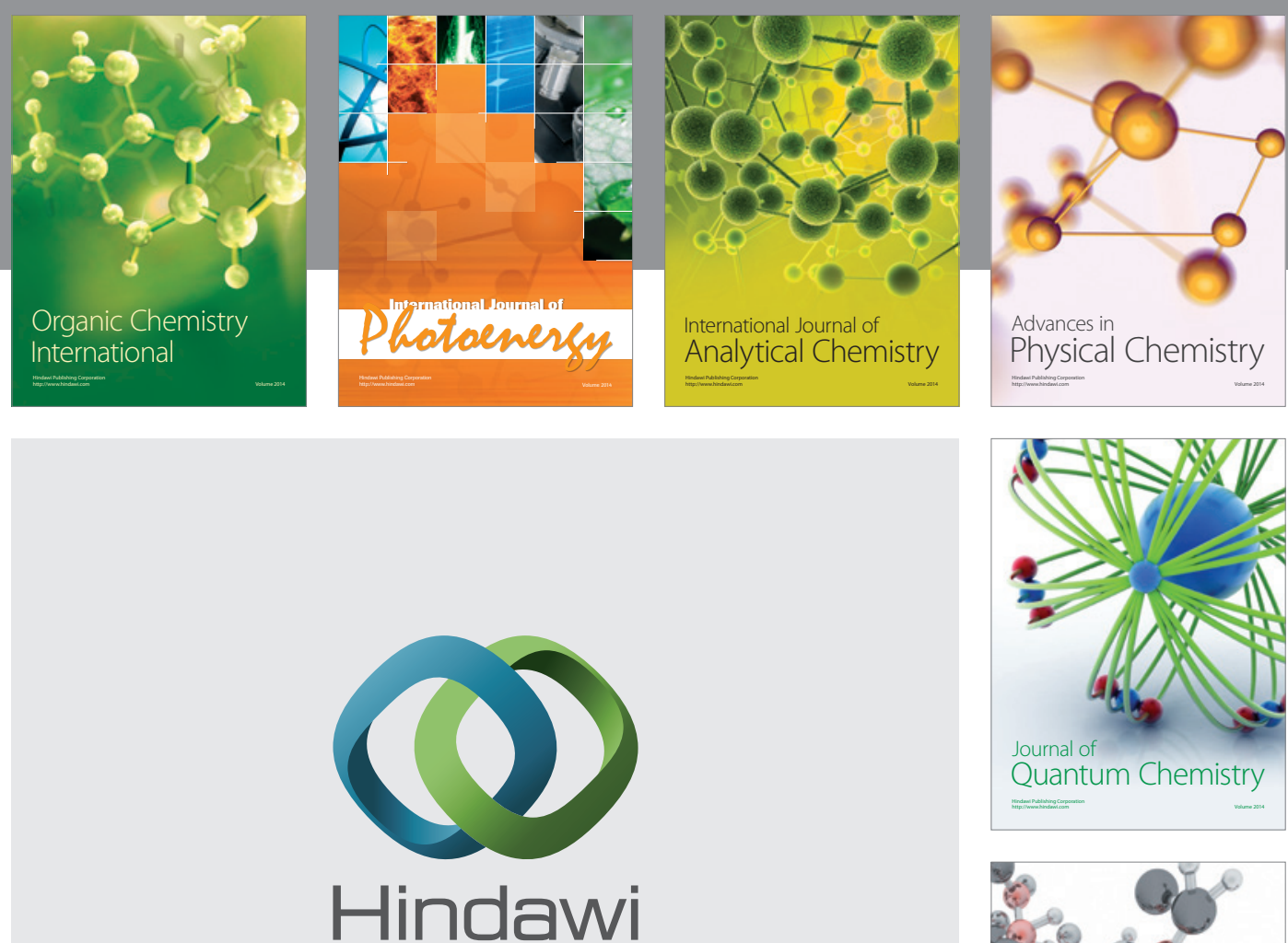

Submit your manuscripts at

http://www.hindawi.com

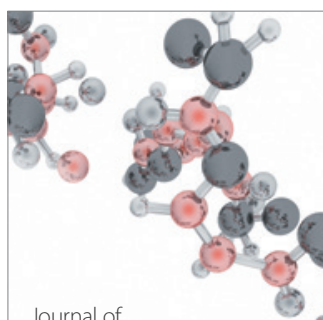

Analytical Methods

in Chemistry

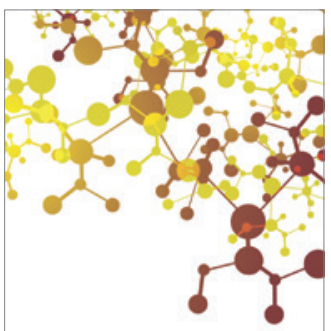

Journal of

Applied Chemistry

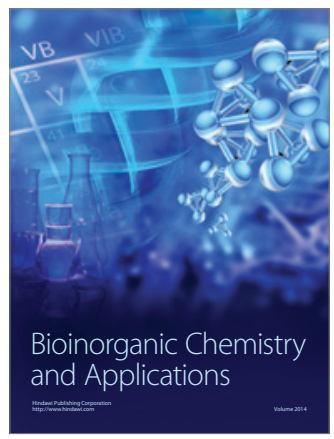

Inorganic Chemistry
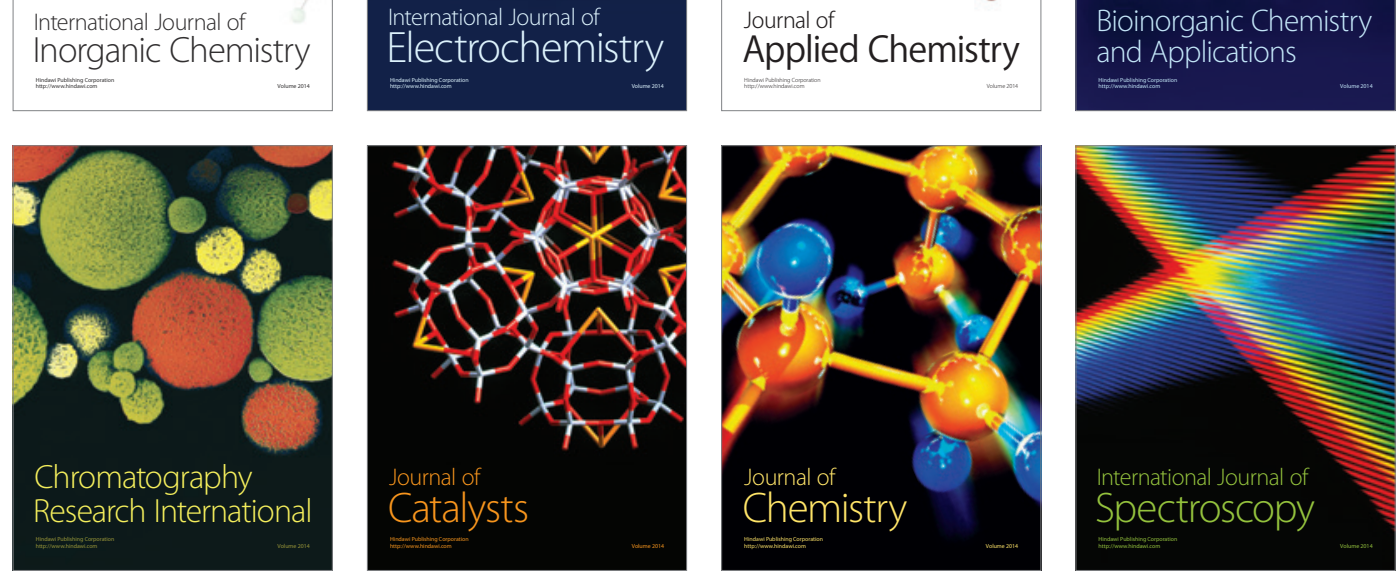